\title{
Nonautonomous Differential Equations in the Algebra of New Generalized Functions
}

\author{
N. V. Bedyuk and O. L. Yablonskii \\ Belarus State University, Minsk, Belarus \\ Received January 8, 2007
}

\begin{abstract}
We study a nonautonomous equation with generalized coefficients in an algebra of generalized functions. The solutions of the equation can be rather different depending on the interpretation of the equation. We show that all these solutions can be obtained from the solution of this equation in the algebra of generalized functions.
\end{abstract}

DOI: $10.1134 /$ S0012266109010029

\section{INTRODUCTION}

The distribution theory developed by Schwartz [1] is one of the main tools for the analysis of solutions of differential equations. However, this theory has an essential drawback, which has been noticed almost from the very beginning. Namely, it is impossible to introduce the product of distributions in a well-defined manner. Therefore, that theory cannot be used in general when analyzing nonlinear differential equations or differential equations with generalized coefficients. Nevertheless, numerous authors suggested various ways of interpreting the solutions of some classes of differential equations with generalized coefficients. Unfortunately, various interpretations of a same equation lead to different solutions, and one can single out a preferred interpretation only with the help of some considerations used when modeling the considered practical problem by that equation.

In the present paper, we consider the differential equation

$$
\dot{x}(t)=f(t, x(t)) \dot{L}(t),
$$

where $\dot{L}(t)$ is the generalized derivative of a function of bounded variation. Consider the main approaches to the interpretation of solutions of this equation. The first approach is related to attempts to analyze this problem in the framework of the theory of generalized functions. The study is focused on the problem of multiplication of discontinuous functions by generalized functions; this problem arises in the expression $f(t, x(t)) \dot{L}(t)$. The definition of the product of a discontinuous function by a generalized function was introduced in $[2 ; 3$, p. 41; 4], and then a solution of the differential equation was constructed. The solutions understood in the sense of various interpretations occurring in these papers do not coincide in general. The second approach involves the formal passage to the integral equation

$$
x(t)=x_{0}+\int_{t_{0}}^{t} f(\xi, x(\xi)) d L(\xi),
$$

where the integral is treated in some sense $[5,6]$. However, with this interpretation, the values of jumps in the solution depend on the definition of the integrand at the points of discontinuity of $L(t)$, which is a disadvantage of this approach. The third approach goes back to the paper [7] and is based on the idea of approximating the desired solution of Eq. (1) by classical solutions induced by smooth approximations to the function $L(t)$. Note that the solutions obtained in [3] with the use of the first and last approaches coincide. 
Yet another approach, which is related to the study of Eq. (1) in the algebra of new generalized functions, is being comprehensively developed nowadays. Some constructions of such algebras were suggested, for example, in $[8,9]$, and a general method for constructing similar algebras was described in [10], where the term "mnemofunction" was used to refer to a new generalized function. The algebra of new generalized random processes, or mnemoprocesses, was constructed in [11] on the basis of the above-mentioned algebras; moreover, the definition of a generalized differential was given there, which proved to be very useful in the analysis of both stochastic differential equations and ordinary differential equations with generalized coefficients from a unified viewpoint (e.g., see $[12-14])$. In the present paper, we treat Eq. (1) as an equation in differentials in the algebra of mnemofunctions and show that, under some conditions, the mnemofunction solution is associated with an ordinary function, which we can naturally call a solution of Eq. (1). In addition, we show that the solution of Eq. (1) in the sense of each of the above-described approaches can be obtained as an associated solution of the equation in differentials in the algebra of mnemofunctions.

\section{MAIN RESULTS}

Suppose that a function $f: \mathbf{T} \times \mathbb{R} \rightarrow \mathbb{R}$, where $\mathbf{T}=[a, b]$, satisfies the Lipschitz condition

$$
\left|f\left(t_{1}, x_{1}\right)-f\left(t_{2}, x_{2}\right)\right|<C_{1}\left|t_{1}-t_{2}\right|+C_{2}\left|x_{1}-x_{2}\right|
$$

and has bounded growth with respect to $x$, i.e., satisfies the inequality

$$
|f(t, x)|<C_{3}(1+|x|)
$$

for all $t \in T$, where $C_{1}, C_{2}$, and $C_{3}$ are constants independent of $t$ and $x$. Let $L: \mathbf{T} \rightarrow \mathbb{R}$ be a right continuous function of bounded variation satisfying the condition $L(a)=0$.

We assume that $L(t)=L(b)$ for $t \geq b$ and $L(t)=L(a)$ for $t \leq a$. To Eq. (1) we assign the following equation in differentials in the algebra of mnemofunctions (for details, see [11, 13, 14]) :

$$
x_{n}\left(t+h_{n}\right)-x_{n}(t)=f_{n}\left(t, x_{n}(t)\right)\left[L_{n}\left(t+h_{n}\right)-L_{n}(t)\right],\left.\quad x_{n}(t)\right|_{\left[a, a+h_{n}\right)}=x_{n}^{0}(t),
$$

where $h_{n}>0 ; L_{n}(t)=\left(L * \varrho_{n}\right)(t)=\int_{0}^{1 / n} L(t+s) \varrho_{n}(s) d s$ with $\varrho_{n} \in C^{\infty}(\mathbb{R}), \varrho_{n} \geq 0, \operatorname{supp} \varrho_{n} \subseteq$ $[0,1 / n]$, and $\int_{0}^{1 / n} \varrho_{n}(s) d s=1 ; f_{n}(t, x)=\left(f * \widetilde{\varrho}_{n}\right)(t, x)=\int_{[0,1 / n]^{2}} f(t+u, x+v) \widetilde{\varrho}_{n}(u, v) d u d v$ with $\widetilde{\varrho}_{n} \in C^{\infty}\left(\mathbb{R}^{2}\right), \widetilde{\varrho}_{n} \geq 0, \operatorname{supp} \widetilde{\varrho}_{n} \subseteq[0,1 / n]^{2}$, and $\int_{[0,1 / n]^{2}} \widetilde{\varrho}_{n}(u, v) d u d v=1$. The initial condition satisfies $x_{n}^{0}(t) \in C^{\infty}\left(\left[a, a+h_{n}\right]\right)$.

For each $t$, we have the representation $t=\tau_{t}+m_{t} h_{n}$, where $\tau_{t} \in\left[a, a+h_{n}\right)$ and $m_{t} \in \mathbb{N}$. Then the solution of problem (4) can be represented in the form

$$
x_{n}(t)=x_{n}\left(\tau_{t}\right)+\sum_{k=0}^{m_{t}-1} f_{n}\left(t_{k}, x_{n}\left(t_{k}\right)\right)\left[L_{n}\left(t_{k+1}\right)-L_{n}\left(t_{k}\right)\right],
$$

where $t_{k}=\tau_{t}+k h_{n}$. Note that the $t_{k}$ depend on $t \in \mathbf{T}$.

In the present paper, we consider the limit behavior of the solution $x_{n}$ of problem (4) as $n \rightarrow \infty$ and $h_{n} \rightarrow 0$. To describe the limit of the sequence $x_{n}$, consider the integral equation

$$
x(t)=x^{0}+\int_{a}^{t} f(s, x(s)) d L^{c}(s)+\sum_{a<s \leq t}(\varphi(\Delta L(s) f(s, \cdot), x(s-), 1)-x(s-)),
$$

where $L^{c}$ is the continuous part of the function $L, \Delta L(s)=L(s+)-L(s-)$ is the value of the jump at a point $s$, and $\varphi$ is the solution of the following integral equation with parameters $z, x$, and $u$ :

$$
\varphi(z, x, u)=x+\int_{[0, u)} z(\varphi(z, x, v)) \mu(d v),
$$


where $z: \mathbb{R} \rightarrow \mathbb{R}$ is some function, $\mu(d v)$ is a probability measure defined on the Borel subsets of the interval $[0,1]$, and the integral is treated in the Lebesgue sense. Throughout the following, we assume that the functions $z$ satisfy the Lipschitz condition; therefore, as shown in [13], Eqs. (7) and (6) have a unique solution.

In what follows, we consider only measures $\mu$ generated by functions $\sigma:[0,1] \rightarrow[0,1]$ of special form. We say that a function $\sigma$ belongs to the class $G$ if there exists an at most countable set of pairwise disjoint intervals $\left(a_{i}, b_{i}\right] \subset[0,1], i \in I$, such that

$$
\sigma(u)=\left\{\begin{array}{lll}
b_{i} & \text { for } & u \in\left(a_{i}, b_{i}\right] \\
u & \text { for } & u \notin \bigcup_{i \in I}\left(a_{i}, b_{i}\right] .
\end{array}\right.
$$

In particular, the set $I$ can be empty.

We introduce a function $F_{n}:[-\infty,+\infty] \rightarrow[0,1]$ by the rule

$$
F_{n}(x)=\int_{x}^{1 / n} \varrho_{n}(s) d s
$$

and the inverse function $F_{n}^{-1}:[0,1] \rightarrow[-\infty,+\infty]$ by the relation

$$
F_{n}^{-1}(u)=\sup \left\{x: F_{n}(x)=u\right\} .
$$

Note that, by definition, $F_{n}^{-1}(0)=+\infty$.

Theorem 1. Let the function $f$ satisfy conditions (2) and (3), let $L$ be a right continuous function of bounded variation, and let $L(a)=0$. Let $\sigma:[0,1] \rightarrow[0,1]$ be a nondecreasing function such that

$$
F_{n}\left(F_{n}^{-1}(u)-\delta h_{n}\right) \rightarrow \sigma(u)
$$

for all points $u \in[0,1]$ of continuity of $\sigma$ and all $\delta \in(0,1)$ as $n \rightarrow \infty$ and $h_{n} \rightarrow 0$. Then $\sigma \in G$ and

$$
\int_{\mathbf{T}}\left|x_{n}(t)-x(t)\right| d t \rightarrow 0
$$

as $n \rightarrow \infty$ and $h_{n} \rightarrow 0$, where $x_{n}$ is the solution of problem (4) and $x$ is the solution of Eq. (6) with measure $\mu$ generated by $\sigma$, provided that

$$
\int_{\mathbf{T}}\left|x_{n}^{0}\left(\tau_{t}\right)-x^{0}\right| d t \rightarrow 0 .
$$

Here $\tau_{t}$ is given by $(5)$.

Remark 1. Thus, by choosing various sequences of "hats" $\varrho_{n}$, we obtain various measures $\mu$, depending on which we arrive at various solutions of Eq. (1). In particular, for an appropriate choice of the sequence $\varrho_{n}$, one can obtain the solutions of Eq. (1) in the sense of all approaches described in Section 1.

\section{AUXILIARY ASSERTIONS}

In what follows, we denote the variation of a function $g$ on the closed interval $[c, d]$ by $V_{c}^{d} g$ and the variation of $g$ on the half-open interval $(c, d]$ by $V_{c+}^{d} g=\lim _{\varepsilon \rightarrow 0+} V_{c+\varepsilon}^{d} g$.

Assertion 1. The following assertions hold for the functions $f_{n}$ and $L_{n}$ occurring in the expression (4).

(i) If $f$ satisfies conditions (2) and (3), then the function $f_{n}$ satisfies the same conditions as well with the same constants.

(ii) $V_{s}^{t} L_{n}=V_{s}^{t+1 / n} L$ and $V_{a}^{b} L_{n}=V_{a}^{b} L$ for all $t, s \in \mathbf{T}, t>s$. 
Proof. The assertion readily follows from the form of the functions $f_{n}$ and $L_{n}$ and the properties of $\varrho_{n}$.

The following lemma provides a generalization of the Gronwall inequality.

Lemma 1 [15]. Let $v$ be a locally bounded measure on $\mathbb{R}$, and let $Y(t)$ be a function of bounded variation on any bounded interval such that

$$
0 \leq Y(t) \leq A+B \int_{[0, t)} Y(s) v(d s)
$$

for any $t \geq 0$, where $A$ and $B$ are nonnegative constants. Then

$$
Y(t) \leq A \exp (B v([0, t))) \prod_{s<t}(1+B v(\{s\})) \exp (-B v(\{s\})) \leq A \exp (B v([0, t)))
$$

for any $t \geq 0$.

By using Lemma 1, one can readily obtain the following estimates for the solution of Eq. (7) (see also [13]).

Assertion 2. Let $\varphi(z, x, u)$ be the solution of Eq. (7) with a function $z$ such that $|z(x)-z(y)| \leq$ $K_{1}|x-y|$ and $|z(x)| \leq K_{2}(1+|x|)$ for any $x, y \in \mathbb{R}$. Then

$$
\begin{aligned}
|\varphi(z, x, u)-x| & \leq K_{2}(|x|+1) \exp \left(K_{2}\right), \\
|\varphi(z, x, u)-x-\varphi(z, y, u)+y| & \leq|x-y| K_{1} \exp \left(K_{1}\right)
\end{aligned}
$$

for any $x, y \in \mathbb{R}$ and $u, v \in[0,1], u>v$.

Assertion 3. Let the function $f$ satisfy conditions (2) and (3), and let $x$ and $x_{n}$ be the solutions of problem (6) and (4), respectively. Then the inequalities

$$
\begin{aligned}
|x(t)| & \leq C\left(1+\left|x^{0}\right|\right), \\
\left|x_{n}(t)\right| & \leq C\left(1+\left|x_{n}^{0}\left(\tau_{t}\right)\right|\right),
\end{aligned}
$$

where $C$ is a constant depending only on $C_{1}, C_{2}, C_{3}$, and $V_{a}^{b} L$, and

$$
\begin{aligned}
|x(t)-x(s)| & \leq C_{3}\left(1+C\left(1+\left|x^{0}\right|\right)\right) V_{s+}^{t} L\left(1+\exp \left(V_{s+}^{t} L C_{3}\right)\right), \\
\left|x_{n}\left(t+l h_{n}\right)-x(t)\right| & \leq C_{3}\left(2+C\left(\left|x_{n}^{0}\left(\tau_{t}\right)\right|+1\right)\right) V_{t}^{t+l h_{n}+1 / n} L
\end{aligned}
$$

hold for any $t, s \in \mathbf{T}, t>s$, and $l, n \in \mathbb{N}$.

Proof. The proof is an obvious modification of that of Lemma 4.5 in [13].

Let us introduce some notation. Since $L$ is a right continuous function of bounded variation, we see that it can be represented in the form $L=L^{c}+L^{d}$, where $L^{c}$ is a continuous function and $L^{d}$ is a piecewise constant right continuous function.

By $\zeta_{i}, i \in \mathbb{N}$, we denote the points of jump discontinuity of the function $L$. Obviously,

$$
L^{d}(t)=\sum_{\zeta_{i} \leq t} \Delta L\left(\zeta_{i}\right)
$$

Moreover, $V_{u}^{v} L=V_{u}^{v} L^{c}+V_{u}^{v} L^{d}$ for any $a \leq u<v \leq b$, and $V_{u}^{v} L^{d}=\sum_{u \leq \zeta_{i} \leq v}\left|\Delta L\left(\zeta_{i}\right)\right|$.

Take an arbitrary $\varepsilon>0$. Since $V_{a}^{b} L<\infty$, we have $V_{a}^{b} L^{d}=\sum_{i=1}^{\infty}\left|\Delta L\left(\zeta_{i}\right)\right|<\infty$. Therefore, there exists an $N \in \mathbb{N}$ such that $\sum_{i=N+1}^{\infty}\left|\Delta L\left(\zeta_{i}\right)\right| \leq \varepsilon$, and $L^{d}$ can be represented in the form

$$
L^{d}=L^{\leq N}+L^{>N}
$$


where

$$
L^{\leq N}(t)=\sum_{i=1}^{N} \mathbb{I}_{\left\{\zeta_{i} \leq t\right\}} \Delta L\left(\zeta_{i}\right), \quad L^{>N}(t)=\sum_{i=N+1}^{\infty} \mathbb{I}_{\left\{\zeta_{i} \leq t\right\}} \Delta L\left(\zeta_{i}\right) .
$$

Then, obviously, $V_{u}^{v} L^{>N} \leq \varepsilon$ and $V_{u}^{v} L^{\leq N} \leq V_{u}^{v} L^{d} \leq \infty$ for any $a \leq u<v \leq b$.

By setting $L_{n}^{c}=L^{c} * \varrho_{n}, L_{n}^{d}=L^{d} * \varrho_{n}, L_{n}^{\leq N}=L^{\leq N} * \varrho_{n}$, and $L_{n}^{>N}=L^{>N} * \varrho_{n}$, we obtain the relations $L_{n}=L_{n}^{c}+L_{n}^{d}$ and $L_{n}^{d}=L_{n}^{\leq N}+L_{n}^{>N}$. Moreover, obviously,

$$
\left|L_{n}^{c}(t)-L^{c}(t)\right| \leq V_{t}^{t+1 / n} L^{c} .
$$

Throughout the following, by $C$ we denote a constant that depends only on $C_{1}, C_{2}, C_{3}, V_{a}^{b} L$, and $|\mathbf{T}|$, where $|\mathbf{T}|=b-a$. This constant can be different in different formulas.

Lemma 2. Let $x$ and $x_{n}$ be the solutions of problem (6) and (4), respectively; then

$$
\begin{aligned}
\mid \sum_{k=0}^{m_{t}-1} f_{n}\left(t_{k}, x_{n}\left(t_{k}\right)\right)[ & \left.L_{n}^{c}\left(t_{k+1}\right)-L_{n}^{c}\left(t_{k}\right)\right]-\int_{a}^{t} f(s, x(s)) d L^{c}(s) \mid \\
\leq & \frac{C}{n}+C\left(1+\left|x_{n}^{0}\left(\tau_{t}\right)\right|+\left|x^{0}\right|\right) \sup _{|u-v| \leq h_{n}+1 / n} V_{u}^{v} L^{c} \\
& +C \sum_{k=0}^{m_{t}-1}\left|x_{n}\left(t_{k}\right)-x\left(t_{k}\right)\right|\left|L^{c}\left(t_{k+1}\right)-L^{c}\left(t_{k}\right)\right| .
\end{aligned}
$$

Proof. Let us rewrite the expression under the sign of absolute value in inequality (16) in the form

$$
\begin{aligned}
& \sum_{k=0}^{m_{t}-1}\left(f_{n}\left(t_{k}, x_{n}\left(t_{k}\right)\right)-f\left(t_{k}, x\left(t_{k}\right)\right)\right)\left[L^{c}\left(t_{k+1}\right)-L^{c}\left(t_{k}\right)\right] \\
& \quad+\sum_{k=0}^{m_{t}-1} f_{n}\left(t_{k}, x_{n}\left(t_{k}\right)\right)\left[\left(L_{n}^{c}\left(t_{k+1}\right)-L_{n}^{c}\left(t_{k}\right)\right)-\left(L^{c}\left(t_{k+1}\right)-L^{c}\left(t_{k}\right)\right)\right] \\
& \quad+\sum_{k=0}^{m_{t}-1} \int_{t_{k}}^{t_{k+1}}\left(f\left(t_{k}, x\left(t_{k}\right)\right)-f(s, x(s))\right) d L^{c}(s)-\int_{a}^{t_{0}} f(s, x(s)) d L^{c}(s)=I_{1}+I_{2}+I_{3}-I_{4} .
\end{aligned}
$$

The definition of $f_{n}$ and the Lipschitz property of $f$ imply the inequality

$$
\left|I_{1}\right| \leq \frac{C}{n}+C \sum_{k=0}^{m_{t}-1}\left|x_{n}\left(t_{k}\right)-x\left(t_{k}\right)\right|\left|L^{c}\left(t_{k+1}\right)-L^{c}\left(t_{k}\right)\right| .
$$

By using summation by parts and by taking into account (11), (13), (15), and the Lipschitz property and bounded growth of $f_{n}$, we obtain the inequality

$$
\begin{aligned}
\left|I_{2}\right| \leq & \left|f_{n}\left(t_{m_{t}-1}, x_{n}\left(t_{m_{t}-1}\right)\right)\left(L_{n}^{c}\left(t_{m_{t}}\right)-L^{c}\left(t_{m_{t}}\right)\right)-f_{n}\left(t_{0}, x_{n}\left(t_{0}\right)\right)\left[L_{n}^{c}\left(t_{0}\right)-L^{c}\left(t_{0}\right)\right]\right| \\
& +\sum_{k=1}^{m_{t}-1}\left|f_{n}\left(t_{k-1}, x_{n}\left(t_{k-1}\right)\right)-f_{n}\left(t_{k}, x_{n}\left(t_{k}\right)\right)\right|\left|L_{n}^{c}\left(t_{k}\right)-L^{c}\left(t_{k}\right)\right| \\
\leq & C\left(1+\left|x_{n}^{0}\left(\tau_{t}\right)\right|\right) \sup _{|v-u|<1 / n} V_{u}^{v} L^{c} .
\end{aligned}
$$


The Lipschitz property of $f$ and inequality (12) imply the estimate

$$
\left|I_{3}\right| \leq C\left(1+\left|x^{0}\right|\right) \sup _{|u-v|<h_{n}} V_{u}^{v} L^{c}
$$

It follows from (3) and (10) that

$$
\left|I_{4}\right|=\left|\int_{a}^{t_{0}} f(s, x(s)) d L^{c}(s)\right| \leq \int_{a}^{t_{0}} C_{3}(1+|x(s)|) d L^{c}(s) \leq C\left(1+\left|x^{0}\right|\right) \sup _{|u-v| \leq h_{n}} V_{u}^{v} L^{c} .
$$

By combining the estimates for $I_{1}-I_{4}$, we obtain inequality (16). The proof of the lemma is complete.

Let $j_{i}, i=1,2, \ldots$, be indices such that $t_{j_{i}} \leq \zeta_{i}-1 / n<t_{j_{i}+1}$; we set $\xi_{k}^{n, i}(t)=F_{n}\left(\zeta_{i}-t_{j_{i}+k}\right)$ for $i=1,2, \ldots, t \in \mathbf{T}, n \in \mathbb{N}, k=0, \ldots, p+2$, and $p=\left[1 /\left(n h_{n}\right)\right]$. Here the brackets stand for the integer part of a number. Note that $\xi_{k}^{n, i}(t)$ depends on $t$, since so do $j_{i}$ and $t_{j_{i}+k}$. One can readily see that $0=\xi_{0}^{n, i}(t) \leq \xi_{1}^{n, i}(t) \leq \cdots \leq \xi_{p+2}^{n, i}(t)=1$; i.e., the $\xi_{k}^{n, i}(t), k=0, \ldots, p+2$, define a partition of the interval $[0,1]$.

For all $n \in \mathbb{N}, x \in \mathbb{R}, i=1,2, \ldots$, and Lipschitz functions $z: \mathbb{R} \rightarrow \mathbb{R}$, we introduce a sequence of functions $\varphi_{k}^{n, i}(z, x, t), t \in \mathbf{T}, k=0, \ldots, p+2$, as follows:

$$
\varphi_{k+1}^{n, i}(t)=\varphi_{k}^{n, i}(t)+z\left(\varphi_{k}^{n, i}(t)\right)\left(\xi_{k+1}^{n, i}(t)-\xi_{k}^{n, i}(t)\right), \quad \varphi_{0}^{n, i}(t)=x .
$$

For all $t \in \mathbf{T}$ and $n=1,2, \ldots$, we define functions of the variable $u \in[0,1]$ by the relations

$$
\begin{aligned}
\sigma_{i}^{n}(u, t) & =\left\{\begin{array}{ccl}
\xi_{k}^{n, i}(t) & \text { if } & \xi_{k-1}^{n, i}(t)<u \leq \xi_{k}^{n, i}(t) \\
0 & \text { if } & u=0
\end{array}\right. \\
\phi^{n, i}(u, t) & =\left\{\begin{array}{ccc}
\varphi_{k}^{n, i}(t) & \text { if } & \xi_{k-1}^{n, i}(t)<u \leq \xi_{k}^{n, i}(t) \\
x & \text { if } & u=0 .
\end{array}\right.
\end{aligned}
$$

Then, in view of the above-introduced notation, we have the identity

$$
\phi^{n, i}(u, t)=x+\int_{[0, u)} z\left(\phi^{n, i}(s, t)\right) \sigma_{i}^{n}(d s, t)
$$

The limit behavior of the sequence $\phi^{n, i}$ was considered in [13, Lemma 5.3]. In view of the conditions described in [16, Lemma 6], one can state this result as follows.

Lemma 3. Let $F_{n}\left(F_{n}^{-1}(u)-\delta h_{n}\right) \rightarrow \sigma(u)$ as $n \rightarrow \infty$ and $h_{n} \rightarrow 0$ for all $\delta \in(0,1)$ and for each point $u \in[0,1]$ of continuity of the function $\sigma$. Then $\sigma$ belongs to the class $G$, and

$$
\int_{\mathbf{T}}\left|\phi^{n, i}(u, t)-\phi(u)\right| d t \rightarrow 0
$$

as $n \rightarrow \infty$ for all $i=1,2, \ldots$ and for any point $u$ of continuity of $\phi$, where $\phi(u)$ is the solution of the equation

$$
\phi(u)=x+\int_{[0, u)} z(\phi(s)) d \sigma(s) .
$$


Lemma 4. For each $\varepsilon>0$, there exists a set $\mathbf{T}_{\varepsilon} \subseteq \mathbf{T}$ and an index $n_{0} \in \mathbb{N}$ such that $\left|\mathbf{T}_{\varepsilon}\right| \leq \varepsilon$ and the inequality

$$
\begin{gathered}
\left|\sum_{k=0}^{m_{t}-1} f_{n}\left(t_{k}, x_{n}\left(t_{k}\right)\right)\left[L_{n}^{d}\left(t_{k+1}\right)-L_{n}^{d}\left(t_{k}\right)\right]-\sum_{\zeta_{i} \leq t}\left[\varphi_{p+2}^{n, i}\left(\Delta L\left(\zeta_{i}\right) f\left(t_{j_{i}}, \cdot\right), x_{n}\left(t_{j_{i}}\right), t\right)-x_{n}\left(t_{j_{i}}\right)\right]\right| \\
\leq C\left(1+\left|x_{n}^{0}\left(\tau_{t}\right)\right|\right)\left(\sup _{|u-v|<1 / n+2 h_{n}} V_{u}^{v} L^{c}+\varepsilon+1 / n\right)
\end{gathered}
$$

holds for all $n>n_{0}$ and $t \in \mathbf{T} \backslash \mathbf{T}_{\varepsilon}$.

Proof. Take an arbitrary $\varepsilon>0$ and find the corresponding $N \in \mathbb{N}$ in the same way as before formula (14). Then there exists an $n_{0} \in \mathbb{N}$ such that $h_{n}+1 / n \leq \min _{0 \leq k<j \leq N}\left|\zeta_{k}-\zeta_{j}\right|$ for all $n>n_{0}$ and $N / n_{0} \leq \varepsilon$. Set $\mathbf{T}_{\varepsilon}=\bigcup_{i=1}^{N}\left(\zeta_{i}-1 / n_{0}, \zeta_{i}\right]$; then $\left|\mathbf{T}_{\varepsilon}\right| \leq \varepsilon$. It follows from the form of $L_{n}^{\leq N}$ that $L_{n}^{\leq N}(t)=L^{\leq N}(t)$ for all $t \notin \bigcup_{i=1}^{N}\left(\zeta_{i}-1 / n, \zeta_{i}\right]$; moreover, $\zeta_{i}$ is the unique point of discontinuity of $L^{\geq N}$ on the interval $\left(\zeta_{i}-1 / n, \zeta_{i}\right]$, and, in addition, $\left(t_{j_{i}}, t_{j_{i}+p+2}\right] \cap\left(t_{j_{r}}, t_{j_{r}+p+2}\right]=\varnothing$ if $i \neq r$. By the definition of $L_{\bar{n}}^{\leq N}$, we have

$$
\begin{aligned}
L_{n}^{\leq N}\left(t_{j_{i}+l+1}\right)-L_{n}^{\leq N}\left(t_{j_{i}+l}\right) & =\Delta L\left(\zeta_{i}\right)\left[F_{n}\left(\zeta_{i}-t_{j_{i}+l+1}\right)-F_{n}\left(\zeta_{i}-t_{j_{i}+l}\right)\right] \\
& =\Delta L\left(\zeta_{i}\right)\left[\xi_{l+1}^{n, i}(t)-\xi_{l}^{n, i}(t)\right]
\end{aligned}
$$

for all $l=0, \ldots, p+2$ and all $i=1,2, \ldots, N$.

By using this representation for $t \in \mathbf{T}_{\varepsilon} \backslash \mathbf{T}$ and $n>n_{0}$, we obtain the relation

$$
\begin{gathered}
\sum_{k=0}^{m_{t}-1} f_{n}\left(t_{k}, x_{n}\left(t_{k}\right)\right)\left[L_{n}^{d}\left(t_{k+1}\right)-L_{n}^{d}\left(t_{k}\right)\right]=\sum_{k=0}^{m_{t}-1} f_{n}\left(t_{k}, x_{n}\left(t_{k}\right)\right)\left[L_{n}^{>N}\left(t_{k+1}\right)-L_{n}^{>N}\left(t_{k}\right)\right] \\
+\sum_{\zeta_{i} \leq t} \sum_{l=0}^{p+1} f_{n}\left(t_{j_{i}+l}, x_{n}\left(t_{j_{i}+l}\right)\right) \Delta L^{\leq N}\left(\zeta_{i}\right)\left[\xi_{l+1}^{n, i}(t)-\xi_{l}^{n, i}(t)\right] .
\end{gathered}
$$

By considering the difference under the sign of absolute value in inequality (19), we obtain

$$
\begin{aligned}
\mid \sum_{k=0}^{m_{t}-1} f_{n}\left(t_{k}, x_{n}\left(t_{k}\right)\right)[ & \left.L_{n}^{d}\left(t_{k+1}\right)-L_{n}^{d}\left(t_{k}\right)\right]-\sum_{\zeta_{i} \leq t}\left[\varphi_{p+2}^{n, i}\left(\Delta L\left(\zeta_{i}\right) f\left(t_{j_{i}}, \cdot\right), x_{n}\left(t_{j_{i}}\right), t\right)-x_{n}\left(t_{j_{i}}\right)\right] \mid \\
\leq & \left|\sum_{k=0}^{m_{t}-1} f_{n}\left(t_{k}, x_{n}\left(t_{k}\right)\right)\left[L_{n}^{>N}\left(t_{k+1}\right)-L_{n}^{>N}\left(t_{k}\right)\right]\right| \\
& +\left|\sum_{\zeta_{i} \leq t}\left[\varphi_{p+2}^{n, i}\left(\Delta L^{>N}\left(\zeta_{i}\right) f\left(t_{j_{i}}, \cdot\right), x_{n}\left(t_{j_{i}}\right), t\right)-x_{n}\left(t_{j_{i}}\right)\right]\right| \\
& +\mid \sum_{\zeta_{i} \leq t} \sum_{l=0}^{p+1} f_{n}\left(t_{j_{i}+l}, x_{n}\left(t_{j_{i}+l}\right)\right) \Delta L^{\leq N}\left(\zeta_{i}\right)\left[\xi_{l+1}^{n, i}(t)-\xi_{l}^{n, i}(t)\right] \\
& -\sum_{\zeta_{i} \leq t}\left[\varphi_{p+2}^{n, i}\left(\Delta L^{\leq N}\left(\zeta_{i}\right) f\left(t_{j_{i}}, \cdot\right), x_{n}\left(t_{j_{i}}\right), t\right)-x_{n}\left(t_{j_{i}}\right)\right] \mid \\
= & I_{1}+I_{2}+I_{3} .
\end{aligned}
$$

Inequalities (3) and (11) and the definition of $L^{>N}$ imply the estimate

$$
I_{1} \leq C\left(\left|x_{n}^{0}\left(\tau_{t}\right)\right|+1\right) \varepsilon
$$


By taking into account (8) and (11), we obtain

$$
I_{2} \leq \varepsilon C\left(1+\left|x_{n}^{0}\left(\tau_{t}\right)\right|\right) .
$$

By the definition of $\varphi_{l}^{n, i}$, we obtain the inequality

$$
\begin{aligned}
I_{3} \leq & \sum_{\zeta_{i} \leq t} \mid \sum_{l=0}^{p+1}\left[f\left(t_{j_{i}}, \varphi_{l}^{n, i}\left(\Delta L^{\leq N}\left(\zeta_{i}\right) f\left(t_{j_{i}}, \cdot\right), x_{n}\left(t_{j_{i}}\right), t\right)\right)\right. \\
& \left.-f_{n}\left(t_{j_{i}+l}, x_{n}\left(t_{j_{i}+l}\right)\right)\right] \Delta L^{\leq N}\left(\zeta_{i}\right)\left[\xi_{l+1}^{n, i}(t)-\xi_{l}^{n, i}(t)\right] \mid .
\end{aligned}
$$

For all $i=1, \ldots, N, t \in \mathbf{T}_{\varepsilon} \backslash \mathbf{T}$, and $l=1, \ldots, p+2$, consider the difference

$$
\begin{aligned}
\mid x_{n}\left(t_{j_{i}+l}\right)- & \varphi_{l}^{n, i}\left(\Delta L^{\leq N}\left(\zeta_{i}\right) f\left(t_{j_{i}}, \cdot\right), x_{n}\left(t_{j_{i}}\right), t\right) \mid \\
\leq & \left|\sum_{k=0}^{l-1} f_{n}\left(t_{j_{i}+k}, x_{n}\left(t_{j_{i}+k}\right)\right)\left[L_{n}^{c}\left(t_{j_{i}+k+1}\right)-L_{n}^{c}\left(t_{j_{i}+k}\right)\right]\right| \\
& +\left|\sum_{k=0}^{l-1} f_{n}\left(t_{j_{i}+k}, x_{n}\left(t_{j_{i}+k}\right)\right)\left[L_{n}^{>N}\left(t_{j_{i}+k+1}\right)-L_{n}^{>N}\left(t_{j_{i}+k}\right)\right]\right| \\
& +\left|\Delta L^{\leq N}\left(\zeta_{i}\right)\right| \sum_{k=0}^{l-1}\left|f_{n}\left(t_{j_{i}+k}, x_{n}\left(t_{j_{i}+k}\right)\right)-f\left(t_{j_{i}}, \varphi_{k}^{n, i}\left(\Delta L^{\leq N}\left(\zeta_{i}\right) f\left(t_{j_{i}}, \cdot\right), x_{n}\left(t_{j_{i}}\right), t\right)\right)\right| \\
& \times\left[\xi_{k+1}^{n, i}(t)-\xi_{k}^{n, i}(t)\right] .
\end{aligned}
$$

By taking into account the form of the functions $f_{n}, L_{n}^{c}$, and $L_{n}^{>N}$, the Lipschitz property and bounded growth of the function $f$, inequality (11), and the definition of $t_{j_{i}}$ and $\xi_{k}^{n, i}(t)$ in the last expression, we obtain

$$
\begin{aligned}
\mid x_{n}\left(t_{j_{i}+l}\right)- & \varphi_{l}^{n, i}\left(\Delta L^{\leq N}\left(\zeta_{i}\right) f\left(t_{j_{i}}, \cdot\right), x_{n}\left(t_{j_{i}}\right), t\right) \mid \\
\leq & C\left(1+\left|x_{n}^{0}\left(\tau_{t}\right)\right|\right)\left(\sup _{|u-v|<1 / n+2 h_{n}} V_{u}^{v} L_{n}^{c}+\varepsilon+1 / n\right) \\
& +\left|\Delta L\left(\zeta_{i}\right)\right| \sum_{k=0}^{l-1}\left|x_{n}\left(t_{j_{i}+k}\right)-\varphi_{k}^{n, i}\left(\Delta L^{\leq N}\left(\zeta_{i}\right) f\left(t_{j_{i}}, \cdot\right), x_{n}\left(t_{j_{i}}\right), t\right)\right|\left[\xi_{k+1}^{n, i}(t)-\xi_{k}^{n, i}(t)\right] .
\end{aligned}
$$

An application of the discrete version of the Gronwall inequality yields

$$
\begin{aligned}
\mid x_{n}\left(t_{j_{i}+k}\right) & -\varphi_{k}^{n, i}\left(\Delta L^{\leq N}\left(\zeta_{i}\right) f\left(t_{j_{i}}, \cdot\right), x_{n}\left(t_{j_{i}}\right), t\right) \mid \\
& \leq C\left(1+\left|x_{n}^{0}\left(\tau_{t}\right)\right|\right)\left(\sup _{|u-v|<1 / n+2 h_{n}} V_{u}^{v} L^{c}+\varepsilon+1 / n\right) .
\end{aligned}
$$

It follows from the last inequality, the estimate (20), the form of $f_{n}$, and the Lipschitz property of the function $f$ that

$$
I_{3} \leq C\left(1+\left|x_{n}^{0}\left(\tau_{t}\right)\right|\right)\left(\sup _{|u-v|<1 / n+2 h_{n}} V_{u}^{v} L^{c}+\varepsilon+1 / n\right) .
$$

By combining the estimates for $I_{1}, I_{2}$, and $I_{3}$, we obtain the desired inequality (19). The proof of the lemma is complete. 


\section{PROOF OF THE MAIN THEOREM}

Recall that the function $f$ satisfies conditions (2) and (3), $L$ is a right continuous function of bounded variation, and $L(a)=0$. In addition,

$$
\int_{t \in \mathbf{T}}\left|x_{n}^{0}\left(\tau_{t}\right)-x^{0}(t)\right| d t \rightarrow 0
$$

and $F_{n}\left(F_{n}^{-1}(u)-\delta h_{n}\right) \rightarrow \sigma(u)$ as $n \rightarrow \infty$ and $h_{n} \rightarrow 0$ for all $\delta \in(0,1)$ and for each point $u \in[0,1]$ of continuity of the monotone function $\sigma$. By $x_{n}$ we denote the solution of problem (4), and by $x$ we denote the solution of problem (6) with measure $\mu(d u)$ generated by the function $\sigma(u)$.

To prove the theorem, we should show that $x_{n}$ tends to $x$ in $L^{1}(\mathbf{T})$. Take an arbitrary $\varepsilon>0$. For this $\varepsilon$, we find $N \in \mathbb{N}$ and represent $L$ in the form $L=L^{c}+L^{\leq N}+L^{>N}$, as defined in (14).

By using the explicit expressions for $x_{n}$ and $x$, we obtain

$$
\begin{aligned}
x_{n}(t)-x(t)= & \left(x_{n}^{0}\left(\tau_{t}\right)-x^{0}\right)+\left(\sum_{k=0}^{m_{t}-1} f_{n}\left(t_{k}, x_{n}\left(t_{k}\right)\right)\left(L^{c}\left(t_{k+1}\right)-L^{c}\left(t_{k}\right)\right)\right. \\
& \left.-\int_{a}^{t} f(s, x(s)) d L^{c}(s)\right)+\left(\sum_{k=0}^{m_{t}-1} f_{n}\left(t_{k}, x_{n}\left(t_{k}\right)\right)\left(L^{d}\left(t_{k+1}\right)-L^{d}\left(t_{k}\right)\right)\right. \\
& \left.-\sum_{a<s \leq t}[\varphi(\Delta L(s) f(s, \cdot), x(s-), 1)-x(s-)]\right)=\left(x_{n}^{0}\left(\tau_{t}\right)-x^{0}\right)+H_{1}+H_{2} .
\end{aligned}
$$

By taking into account the estimate (16), we obtain the inequality

$$
\begin{aligned}
\left|H_{1}\right| \leq & C / n+C\left(1+\left|x_{n}^{0}\left(\tau_{t}\right)\right|+\left|x^{0}\right|\right) \sup _{|u-v| \leq h_{n}+1 / n} V_{u}^{v} L^{c} \\
& +C \sum_{k=0}^{m_{t}-1}\left|x_{n}\left(t_{k}\right)-x\left(t_{k}\right)\right|\left|L^{c}\left(t_{k+1}\right)-L^{c}\left(t_{k}\right)\right| .
\end{aligned}
$$

By using the notation $\varphi_{k}^{n, i}$ and $j_{i}$ introduced in (17), we arrive at the inequality

$$
\begin{aligned}
\left|H_{2}\right| \leq & \mid \sum_{k=0}^{m_{t}-1} f_{n}\left(t_{k}, x_{n}\left(t_{k}\right)\right)\left(L^{d}\left(t_{k+1}\right)-L^{d}\left(t_{k}\right)\right) \\
& -\sum_{\zeta_{i} \leq t}\left[\varphi_{p+2}^{n, i}\left(\Delta L\left(\zeta_{i}\right) f\left(t_{j_{i}}, \cdot\right), x_{n}\left(t_{j_{i}}\right), t\right)-x_{n}\left(t_{j_{i}}\right)\right] \mid \\
& +\left|\sum_{\zeta_{i} \leq t}\left[\varphi_{p+2}^{n, i}\left(\Delta L^{>N}\left(\zeta_{i}\right) f\left(t_{j_{i}}, \cdot\right), x_{n}\left(t_{j_{i}}\right), t\right)-x_{n}\left(t_{j_{i}}\right)\right]\right| \\
& +\mid \sum_{\zeta_{i} \leq t}\left[\left(\varphi_{p+2}^{n, i}\left(\Delta L^{\leq N}\left(\zeta_{i}\right) f\left(t_{j_{i}}, \cdot\right), x_{n}\left(t_{j_{i}}\right), t\right)\right)-x_{n}\left(t_{j_{i}}\right)\right) \\
& \left.-\left(\varphi_{p+2}^{n, i}\left(\Delta L^{\leq N}\left(\zeta_{i}\right) f\left(t_{j_{i}}, \cdot\right), x\left(t_{j_{i}}\right), t\right)-x\left(t_{j_{i}}\right)\right)\right] \mid \\
& +\mid \sum_{\zeta_{i} \leq t}\left[\left(\varphi_{p+2}^{n, i}\left(\Delta L^{\leq N}\left(\zeta_{i}\right) f\left(t_{j_{i}}, \cdot\right), x\left(t_{j_{i}}\right), t\right)-x\left(t_{j_{i}}\right)\right)\right. \\
& \left.-\left(\varphi_{p+2}^{n, i}\left(\Delta L^{\leq N}\left(\zeta_{i}\right) f\left(\zeta_{i}, \cdot\right), x\left(\zeta_{i}-\right), t\right)-x\left(\zeta_{i}-\right)\right)\right] \mid
\end{aligned}
$$




$$
\begin{aligned}
& +\mid \sum_{\zeta_{i} \leq t}\left[\left(\varphi_{p+2}^{n, i}\left(\Delta L^{\leq N}\left(\zeta_{i}\right) f\left(\zeta_{i}-, \cdot\right), x\left(\zeta_{i}-\right), t\right)\right.\right. \\
& \left.\left.-x\left(\zeta_{i}-\right)\right)-\left(\varphi\left(\Delta L^{\leq N}\left(\zeta_{i}\right) f\left(\zeta_{i}-, \cdot\right), x\left(\zeta_{i}-\right), t\right)-x\left(\zeta_{i}-\right)\right)\right] \mid \\
& +\left|\sum_{a<s \leq t}\left(\varphi\left(\Delta L^{>N}(s) f(s, \cdot), x(s-), t\right)-x(s-)\right)\right| \\
& =S_{1}+S_{2}+S_{3}+S_{4}+S_{5}+S_{6} .
\end{aligned}
$$

Let us estimate each term. Just as above, we assume that $C$ is a constant depending only on $|\mathbf{T}|$, $V_{a}^{b} L, C_{1}, C_{2}$, and $C_{3}$. Then, by Lemma 4 , we find a set $\mathbf{T}_{\varepsilon}$ and an index $n_{0}$ such that

$$
\left.\left|S_{1}\right| \leq C\left(1+\left|x_{n}^{0}\left(\tau_{t}\right)\right|\right)\right)\left(\sup _{|u-v|<1 / n+2 h_{n}} V_{u}^{v} L^{c}+\varepsilon+1 / n\right)
$$

for all $n>n_{0}$ and $t \in \mathbf{T} \backslash \mathbf{T}_{\varepsilon}$.

Just as for the term $I_{2}$ in Lemma 4, we have

$$
\left|S_{2}\right| \leq C \varepsilon\left(1+\left|x_{n}^{0}\left(\tau_{t}\right)\right|\right)
$$

It follows from (8) and (10) that

$$
\left|S_{6}\right| \leq C \varepsilon\left(1+\left|x^{0}\right|\right)
$$

By applying the estimate (9) to $S_{3}$, we obtain

$$
\left|S_{3}\right| \leq C \sum_{i=1}^{N}\left|\Delta L\left(\zeta_{i}\right)\right|\left|x_{n}\left(t_{j_{i}}\right)-x\left(t_{j_{i}}\right)\right|
$$

By proceeding in a similar way and by using inequality (12), we obtain the estimate

$$
\left|S_{4}\right| \leq C \sum_{i=1}^{N}\left|\Delta L\left(\zeta_{i}\right)\right|\left|x\left(t_{j_{i}}\right)-x\left(\zeta_{i}-\right)\right| \leq C\left(1+\left|x^{0}\right|\right) V_{t_{j_{i}}}^{\zeta_{i}-} L .
$$

By the definition of $t_{j_{i}}$ and $n_{0}$, we have $V_{t_{j_{i}}}^{\zeta_{i}-} L \leq V_{t_{j_{i}}}^{\zeta_{i}} L^{c}+\varepsilon$ for all $i=1, \ldots, N$. Consequently,

$$
\left|S_{4}\right| \leq C\left(1+\left|x^{0}\right|\right)\left(\sup _{|u-v|<1 / n+h_{n}} V_{u}^{v} L^{c}+\varepsilon\right)
$$

Consider the term $S_{5}$ :

$$
\left|S_{5}\right| \leq \sum_{i=1}^{N}\left|\varphi_{p+2}^{n, i}\left(\Delta L^{\leq N}\left(\zeta_{i}\right) f\left(\zeta_{i}, \cdot\right), x\left(\zeta_{i}-\right), t\right)-\varphi\left(\Delta L^{\leq N}\left(\zeta_{i}\right) f\left(\zeta_{i}, \cdot\right), x\left(\zeta_{i}-\right), t\right)\right|=\sum_{i=1}^{N} z_{n}^{i, N}(t) .
$$

By substituting the resulting estimates into (21), we obtain

$$
\begin{aligned}
\left|H_{2}\right| \leq & C\left(1+\left|x^{0}\right|+\left|x_{n}^{0}\left(\tau_{t}\right)\right|\right)\left[\sup _{|u-v|<2 / n} V_{u}^{v} L^{c}+\varepsilon+\frac{1}{n}\right] \\
& +C \sum_{i=1}^{N}\left|\Delta L\left(\zeta_{i}\right)\right|\left|x_{n}\left(t_{j_{i}}\right)-x\left(t_{j_{i}}\right)\right|+\sum_{i=1}^{N} z_{n}^{i, N}(t) .
\end{aligned}
$$

DIFFERENTIAL EQUATIONS $\quad$ Vol. $45 \quad$ No. $1 \quad 2009$ 
By using the estimates for $H_{1}$ and $H_{2}$, we obtain the inequality

$$
\begin{aligned}
\left|x_{n}(t)-x(t)\right| \leq & \left|x_{n}^{0}\left(\tau_{t}\right)-x^{0}\right|+C \sum_{k=0}^{m_{t}-1}\left|x_{n}\left(t_{k}\right)-x\left(t_{k}\right)\right|\left|L^{c}\left(t_{k+1}\right)-L^{c}\left(t_{k}\right)\right|+\sum_{i=1}^{N} z_{n}^{i, N}(t) \\
& +C\left(1+\left|x^{0}\right|+\left|x_{n}^{0}\left(\tau_{t}\right)\right|\right)\left[\sup _{|u-v|<2 h_{n}+2 / n} V_{u}^{v} L^{c}+\varepsilon+\frac{1}{n}\right] \\
& +C \sum_{i=1}^{N}\left|\Delta L\left(\zeta_{i}\right)\right|\left|x_{n}\left(t_{j_{i}}\right)-x\left(t_{j_{i}}\right)\right| .
\end{aligned}
$$

By applying the discrete Gronwall inequality to the last inequality, we obtain

$$
\begin{aligned}
\left|x_{n}(t)-x(t)\right| \leq & C\left|x_{n}^{0}\left(\tau_{t}\right)-x^{0}\right|+C\left(1+\left|x_{n}^{0}\left(\tau_{t}\right)\right|+\left|x^{0}\right|\right)\left[\sup _{|u-v|<2 h_{n}+2 / n} V_{u}^{v} L^{c}+\varepsilon+\frac{1}{n}\right] \\
& +C \sum_{i=1}^{N} z_{n}^{i, N}(t)
\end{aligned}
$$

for all $n>n_{0}$ and $t \in \mathbf{T} \backslash \mathbf{T}_{\varepsilon}$.

By integrating both sides of inequality (22) over $\mathbf{T} \backslash \mathbf{T}_{\varepsilon}$ and by taking into account the inequalities $\left|\mathbf{T}_{\varepsilon}\right| \leq \varepsilon,(10)$, and (11), we obtain

$$
\begin{aligned}
\int_{\mathbf{T}}\left|x_{n}(t)-x(t)\right| d t \leq & C \int_{\mathbf{T}}\left|x_{n}^{0}\left(\tau_{t}\right)-x^{0}\right| d t+C \sum_{i=1}^{N} \int_{\mathbf{T}} z_{n}^{i, N}(t) d t \\
& +C\left(1+\left|x^{0}\right|+\int_{\mathbf{T}}\left|x_{n}^{0}\left(\tau_{t}\right)\right| d t\right)\left[\sup _{|u-v| \leq h_{n}+1 / n} V_{u}^{v} L^{c}+\varepsilon+\frac{1}{n}\right] .
\end{aligned}
$$

By the assumption of the theorem, $\int_{\mathbf{T}}\left|x_{n}^{0}\left(\tau_{t}\right)-x^{0}\right| d t \rightarrow 0$. Therefore, $\int_{\mathbf{T}}\left|x_{n}^{0}\left(\tau_{t}\right)\right| d t \leq C$ for any $n \in \mathbb{N}$. Since $V_{a}^{t} L^{c}$ is a continuous function on the closed interval $\mathbf{T}$, we find that it is equicontinuous on it. Therefore,

$$
\sup _{|u-v| \leq 2 h_{n}+2 / n} V_{u}^{v} L^{c} \rightarrow 0 \quad \text { as } \quad n \rightarrow \infty, \quad h_{n} \rightarrow 0 .
$$

Recall that $\varphi_{k}^{n, i}(z, x, t)$ is given by (17) and the functions $\sigma_{i}^{n}(u, t)$ are defined in (18). By the assumption of the theorem, $F_{n}\left(F_{n}^{-1}(u)-\delta h_{n}\right) \rightarrow \sigma(u)$ as $n \rightarrow \infty$ and $h_{n} \rightarrow 0$ for all $\delta \in(0,1)$ and for each point $u \in[0,1]$ of continuity of the function $\sigma$. Then it follows from Lemma 3 that $\sigma$ belongs to the class $G$ and $\int_{\mathrm{T}} z_{n}^{i, N}(t) d t \rightarrow 0$ as $n \rightarrow \infty$ and $h_{n} \rightarrow 0$ for all $1 \leq i \leq N$.

By letting $n$ tend to infinity and $h_{n}$ to zero in (23), we obtain

$$
\limsup _{n \rightarrow \infty, h_{n} \rightarrow 0} \int_{\mathbf{T}}\left|x_{n}(t)-x(t)\right| d t \leq C \varepsilon,
$$

which, together with the arbitrary choice of $\varepsilon$, implies that

$$
\int_{\mathbf{T}}\left|x_{n}(t)-x(t)\right| d t \rightarrow 0 \quad \text { as } \quad n \rightarrow \infty, \quad h_{n} \rightarrow 0 .
$$

The proof of the theorem is complete. 


\section{REFERENCES}

1. Schwartz, L., Théorie des distributions. I, II, Paris: Hermann, 1950, 1951.

2. Antosik, P. and Liegza, J., in Generalized Functions and Operational Calculus: Proc. Conf., Varna, 1979 , pp. 20-26.

3. Zavalishchin, S.T. and Sesekin, A.N., Impul'snye protsessy. Modeli i prilozheniya (Impulse Processes: Models and Applications), Moscow: Nauka, 1991.

4. Derr, V. and Kinzebulatov, D., Distributions with Dynamic Test Functions and Multiplication by Discontinuous Functions, Preprint, arXiv:math.CA/0603351, 2006.

5. Das, P.C. and Sharma, R.R., Czechoslovak Math. J., 1972, vol. 22, no. 1, pp. 145-158.

6. Pandit, S.G. and Deo, S.G., Lecture Notes in Math., 1982, vol. 954.

7. Kurzweil, J., Czechoslovak Math. J., 1958, vol. 8, no. 1, pp. 360-388.

8. Colombeau, J.F., Elementary Introduction to New Generalized Functions, Amsterdam: North-Holland, 1985.

9. Egorov, Yu.V., Uspekhi Mat. Nauk, 1990, vol. 45, no. 5(275), pp. 3-40.

10. Antonevich, A.B. and Radyno, Ya.V., Dokl. Akad. Nauk SSSR, 1991, vol. 318, no. 2, pp. 267-270.

11. Lazakovich, N.V., Dokl. Akad. Nauk Belarusi, 1994, vol. 38, no. 5, pp. 23-27.

12. Lazakovich, N.V. and Yablonski, A.L., Stoch. Stoch. Rep., 2004, vol. 76, no. 2, pp. 135-145.

13. Yablonski, A., Nonlinear Anal., 2005, vol. 63, pp. 171-197.

14. Koval'chuk, A.N., Novokhrost, V.G., and Yablonskii, O.L., Izv. Vyssh. Uchebn. Zaved. Mat., 2005, no. 3, pp. 23-31.

15. Groh, J., Illinois J. Math., 1980, vol. 24 (2), pp. 244-263.

16. Lazakovich, N.V. and Yablonskii, O.L., Teor. Veroyatnost. Primenen., 2005, vol. 50, no. 4, pp. 711-732. 